\title{
Erratum to: Strategies for treatment of dystonia
}

\author{
Dirk Dressler $^{1} \cdot$ Eckart Altenmueller $^{2} \cdot$ Roongroj Bhidayasiri $^{3} \cdot$ Saeed Bohlega $^{4} \cdot$ \\ Pedro Chana ${ }^{5}$ - Tae Mo Chung ${ }^{6} \cdot$ Steven Frucht $^{7} \cdot$ Pedro J. Garcia-Ruiz ${ }^{8}$. \\ Alain Kaelin ${ }^{9} \cdot$ Ryuji Kaji ${ }^{10}$ - Petr Kanovsky ${ }^{11}$ - Rainer Laskawi ${ }^{12}$. \\ Federico Micheli ${ }^{13} \cdot$ Olga Orlova $^{14} \cdot$ Maja Relja $^{15} \cdot$ Raymond Rosales $^{16}$. \\ Jaroslaw Slawek $^{17} \cdot$ Sofia Timerbaeva $^{18} \cdot$ Thomas T. Warner $^{19} \cdot$ Fereshte Adib Saberi $^{20}$
}

Published online: 6 November 2015

(c) Springer-Verlag Wien 2015

\section{Erratum to: J Neural Transm DOI 10.1007/s00702-015-1453-x}

The author would like to correct the errors in the publication of the original article. The corrected details are given below for your reading.

In the original article, one of the co-author's (Saeed Bohlega) family name has been published incorrectly. The correct family name should be Bohlega.

The online version of the original article can be found under doi:10.1007/s00702-015-1453-x.

Dirk Dressler

dressler.dirk@mh-hannover.de

1 Department of Neurology, Hannover Medical School, Carl-Neuberg-Str. 1, D-30625 Hannover, Germany

2 Institute of Music Physiology and Musicians' Medicine, Hanover University of Music, Drama and Media, Hannover, Germany

3 Chulalongkorn Centre for Excellence on Parkinson's Disease and Related Disorders, King Chulalongkorn Memorial Hospital, Bangkok, Thailand

4 Department of Neurology, King Faisal Specialist Hospital, Riyadh, Kingdom of Saudi Arabia

5 Centro de Estudios de Trastornos del Movimiento, University of Santiago, Santiago de Chile, Chile

6 Hospital Sao Joaquim Beneficencia Portuguesa, Sao Paulo, Brazil

7 Department of Neurology, Mount Sinai Medical Center, New York, NY, USA

8 Department of Neurology, Fundacion Jimenez Diaz, Madrid, Spain

9 Neurocentro della Svizzera Italiana, Ospedale Regionale di Lugano, Lugano, Switzerland
The affiliation of the Jaroslaw Slawek is incorrect. The correct affiliation is:

Department of Neurological-Psychiatric Nursing, Medical University of Gdansk, Gdansk, Poland.

The original article has also been updated.

10 Department of Neurology, University of Tokushima, Tokushima, Japan

11 Department of Neurology, Palacky University, Olomouc, Czech Republic

12 Department of Ear-, Nose and Throat, Universitätsmedizin Göttingen, Göttingen, Germany

13 Department of Neurology, Hospital de Clínicas José de San Martín, University of Buenos Aires, Buenos Aires, Argentina

14 Clinic 'Cecil Plus', Moscow, Russia

15 Department of Neurology, University of Zagreb, Zagreb, Croatia

16 Department of Neurology, University of Santo Tomas, Manila, Philippines

17 Department of Neurological-Psychiatric Nursing, Medical University of Gdansk, Gdansk, Poland

18 Scientific Research Institute of Neurology, Moscow, Russia

19 Reta Lila Weston Institute, University College, London, UK

20 IAB-Interdisciplinary Working Group for Movement Disorders, Hamburg, Germany 